\title{
Role of Alexithymia in Predicting Self-Efficacy in Academic Students of a North Indian Institution: A Cross Sectional Study
}

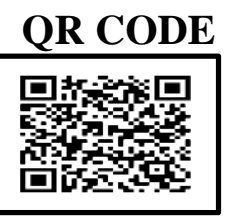

DEVANSHU CHAUDHARY, ANJALI MEENA*2

INTRODUCTION: Alexithymia is a cognitive-affective style and a personality construct that is associated with various physical and psychological disorders. It refers to a personality trait associated with difficulties with identifying or describing feelings, mental imaging and fantasy, and external cognitive orientation

AIM: To access the problem of alexithymia among the Academic (Dental and Ayurvedic) students of an Institution in North India.

MATERIALS AND METHOD: A questionnaire based cross-sectional survey was carried out among the medical students of D.J. Group of Institutions, Modinagar, Ghaziabad district, Uttar Pradesh, India. Ethical approval was obtained from the Institutional Review Board, Modinagar and informed consent was obtained from all the study participants prior to the study.

RESULTS: The questionnaire based study was carried out among 252 medical students of D.J. Group of Institutions, Modinagar, Ghaziabad district, Uttar Pradesh, India. Out of which 113 students were of Ayurveda and 139 were dental students. The inter group comparison of respondents according to course of study in which majority of the Ayurveda students were having significant result for the alexithymia problem.

CONCLUSION: This study demonstrates that alexithymia plays significant role in decreasing self-efficacy in academic students. As alexithymia is high in college students and affects self-regulation, goal orientation, and academic function, we suggest that mental physicians routinely evaluate medical college students for alexithymia.

KEYWORDS: Alexithymia, Ayurveda, Dental, Student

\section{INTRODUCTION}

Now a day's people are facing difficulties in identifying, describing, and expressing their subjective feelings and cannot distinguish between feelings and the emotional arousal. Even they have difficulty in reflecting and regulating their emotions with constricted imagination capacities such as paucity of fantasies, and have an externally oriented cognitive thinking, which is popularly known as Alexithymia. Literally, alexithymia is a Greek word meaning "lack of words for emotions" or lack of the ability to find words that identify and describe feelings. Alexithymia is a cognitive-affective style and a personality construct that is associated with various physical and psychological disorders. ${ }^{1}$ Also; it is difficult for people with alexithymia to verbally communicate their psychological distress, with failure to seek help from others. As a result, this could lead to a feeling of isolation and not being understood by others and potential of negative effect. ${ }^{2}$ It is associated with a trait of human personality that has difficulties with identifying or describing feelings, mental imaging and fantasy, and external cognitive orientation. ${ }^{1}$

Researches have also documented that alexithymia is often found to be associated with certain psychiatric disorders, such as psychosomatic disorders ${ }^{2-4}$, depression $^{5}$, and anxiety. ${ }^{6} \mathrm{~A}$ few studies have reported a positive relationship between alexithymia and pain disorders in their study population. ${ }^{7,8}$ It has the tendency to influence the academic achievement of college students, and that effect could also be moderate in nature. ${ }^{9}$ A positive relationship between test anxiety and alexithymia among students was reported by and Sepahvand et al. (2015). ${ }^{10}$

The prevalence of different kinds of psychiatric disorders are more frequently observed among college students than in a matched non-student populations and this reason could be due to multiple stressors, which include, but are not limited to academic overload, inadequate time, final examinations and peer pressure. ${ }^{11,12}$ The above statement can be supported as there is documented evidence that a marked increase in psychiatric disorders, including depression and anxiety in college students has been seen over the last decade. ${ }^{13}$

Authors across the world have reported a high degree of alexithymia in medical students ${ }^{8,14}$ and subsequently, its influence on their mental well being. ${ }^{15}$ Among students, low self-efficacy was associated with depression and anxiety. ${ }^{16}$ However, there still are numerous gaps in the academic mental health literature and the questions among the scientific 
community is that (i) whether low self-efficacy is associated with alexithymia, (ii)whether alexithymia is related to depression and anxiety in academic students, and (iii) which factors impact alexithymia in academic students and hence, this study was undertaken with an aim to provide the scientific community with important data regarding the problem of alexithymia among the medical students.

\section{MATERIALS \& METHOD}

A questionnaire based cross-sectional survey was carried out among the medical students of D.J. Group of Institutions, Modinagar, Ghaziabad district, Uttar Pradesh, India. Ethical approval was obtained from the Institutional Review Board, Modinagar and informed consent was obtained from all the study participants prior to the study.

\section{DATA COLLECTION}

A total of 252 students were included in the study and those who were non-hostellers was excluded. These students were divided into 2 groups. One group having dental students whereas other one includes ayurveda students.

The questionnaire used in the study consisted of 20 Questions. Face validity and content validity was assessed. Reliability of the questionnaire was assessed using test-retest and internal consistency of the questionnaire was ascertained by Chronbachs-Alpha $(\alpha)$. Construct validity of the questionnaire was assessed using Spearman's correlation coefficient between individual parameter/construct and overall score of the construct. The data was entered and analyzed in SPSS version 16.o. and both descriptive and inferential tests were applied.

\section{RESULTS}

The questionnaire based study was carried out among 252 academic students of D.J. Group of Institutions, Modinagar, Ghaziabad district, Uttar Pradesh, India. Out of which 113 students were of Ayurveda and 139 were dental students. Table 1 shows the frequency distribution of respondent regarding alexithymia in which majority of the respondents were agreeing regarding the alexithymia problem whereas table 2 shows the inter group comparison of respondents according to course of study in which majority of the Ayurveda students were having significant result for the alexithymia problem.

\section{DISCUSSION}

The condition of alexithymia is referred to a personality trait associated with difficulties with identifying or describing feelings, mental imaging and fantasy, and external cognitive orientation in a person. This construct was first conceptualized by Nemiah and Sifneos (1970) as a difficulty in the communicative patterns of certain individuals who demonstrated an inability to verbally express or describe feelings, as well as had a marked diminution of fantasy and since then, many researchers have tried to test and expand this construct. As per Bagby, Taylor, Parker \& Dickens, (2006), ${ }^{7}$ alexithymia is a disorder of affect regulation resulting from developmental impairments in cognitive representations that process all emotional information.

The present questionnaire based study which consisted of questions regarding the perception of the study participants towards the alexithymia scale. The study was carried out among the academic students which consist of ayurveda students and dental students where it was clearly seen that majority of the participants did agree and some did strongly agree when they were asked wether the found it difficult to say how they felt inside or about expressing the exact feeling of anger, being scared and/or sad and it highlights a majority of the students academic students in the dental and ayurvedic filed are suffering from the problem of alexithymia. In agreement, few authors have also reported a positive relationship between alexithymia and pain disorders among students. ${ }^{8-10}$

\section{CONCLUSION}

This study demonstrates that alexithymia plays significant role in decreasing self-efficacy in academic students. As alexithymia is high in college students and affects self-regulation, goal orientation, and academic function it is suggested that they are regularly assessed and easy access to college counsellors and support groups is available, especially during examinations.

\section{REFERENCES}

1. Taylor GJ, Bagby RM, Kushner SC, Benoit D, Atkinson L. Alexithymia and adult attachment representations: associations with the five-factor model of personality and perceived relationship adjustment. Comprehensive Psychiatry 2014; 55(5):1258-68.

2. Faramarzi M, Azadfallah P, Book HE, Tabatabaei KR, Taheri H, Shokri-shirvani J. A randomized controlled 


\begin{tabular}{|c|c|c|c|c|c|c|}
\hline QUESTION & $\begin{array}{l}\text { STRONGLY } \\
\text { AGREE } \\
\text { n (\%) }\end{array}$ & $\begin{array}{l}\text { AGREE } \\
\text { n (\%) }\end{array}$ & $\begin{array}{c}\text { NEITHER } \\
\text { AGREE NOR } \\
\text { DISAGREE } \\
\text { n (\%) }\end{array}$ & $\begin{array}{c}\text { DISAGREE } \\
\text { n (\%) }\end{array}$ & $\begin{array}{c}\text { STRONGLY } \\
\text { DISAGREE } \\
\text { n (\%) }\end{array}$ & $\begin{array}{l}\text { MEAN } \pm \\
\text { SD }\end{array}$ \\
\hline $\begin{array}{c}\text { 1. I am often } \\
\text { confused about the } \\
\text { way I am feeling } \\
\text { inside }\end{array}$ & o $(0 \%)$ & $169(67.1 \%)$ & $30(11.9 \%)$ & $53(21.0 \%)$ & $\mathrm{o}(\mathrm{o} \%)$ & $\begin{array}{l}3 \cdot 46 \pm \\
0.820\end{array}$ \\
\hline $\begin{array}{l}\text { 2. I find it difficult to } \\
\text { say how I feel inside }\end{array}$ & o $(0 \%)$ & 169(67.1\%) & $30(11.9 \%)$ & $53(21.0 \%)$ & $\mathrm{o}(\mathrm{o} \%)$ & $\begin{array}{l}3 \cdot 46 \pm \\
0.820\end{array}$ \\
\hline $\begin{array}{l}\text { 3. I feel things in my } \\
\text { body that even } \\
\text { doctors don't } \\
\text { understand }\end{array}$ & $3(1.2 \%)$ & $145(57 \cdot 5 \%)$ & $36(14.3 \%)$ & $68(27.0 \%)$ & o $(\mathrm{o} \%)$ & $\begin{array}{l}3 \cdot 33 \pm \\
0.887\end{array}$ \\
\hline $\begin{array}{l}\text { 4. I can easily say } \\
\text { how I feel inside }\end{array}$ & $16(6.3 \%)$ & $169(67.1 \%)$ & $34(13.5 \%)$ & $33(13.1 \%)$ & o $(0 \%)$ & $\begin{array}{l}3.67 \pm \\
0.783\end{array}$ \\
\hline $\begin{array}{l}\text { 5. When I have a } \\
\text { problem, I want to } \\
\text { know where it comes } \\
\text { from and not just } \\
\text { talk about it }\end{array}$ & $30(11.9 \%)$ & $147(58.3 \%)$ & $44(17 \cdot 5 \%)$ & $26(10.3 \%)$ & $5(2.0 \%)$ & $\begin{array}{l}3.68 \pm \\
0.886\end{array}$ \\
\hline $\begin{array}{l}\text { 6. When I am upset, I } \\
\text { don't know if I am } \\
\text { sad, scared or angry }\end{array}$ & $17(6.7 \%)$ & $157(62.3 \%)$ & $41(16.3 \%)$ & $37(14.7 \%)$ & o $(0 \%)$ & $3.61 \pm 0.818$ \\
\hline $\begin{array}{l}\text { 7. I am often puzzled } \\
\text { by things that I feel } \\
\text { in my body } \\
\end{array}$ & $9(3.6 \%)$ & $144(57.1 \%)$ & $17(6.7 \%)$ & $79(31.3 \%)$ & $3(1.2 \%)$ & $3.31 \pm 0.993$ \\
\hline $\begin{array}{l}\text { 8. I'd rather wait and } \\
\text { see what happens, } \\
\text { instead of thinking } \\
\text { about why things } \\
\text { happen }\end{array}$ & $\mathrm{o}(\mathrm{o} \%)$ & $126(50.0 \%)$ & $61(24.2 \%)$ & $65(25.8 \%)$ & o $(0 \%)$ & $\begin{array}{l}3.24 \pm \\
0.838\end{array}$ \\
\hline $\begin{array}{l}\text { 9. Sometimes I can't } \\
\text { find the words to say } \\
\text { how I feel inside }\end{array}$ & $37(14.7 \%)$ & $150(59.5 \%)$ & $45(17.9 \%)$ & $20(7 \cdot 9 \%)$ & o $(0 \%)$ & $3.81 \pm 0.780$ \\
\hline $\begin{array}{c}\text { 10. It is important to } \\
\text { understand how you } \\
\text { feel inside }\end{array}$ & $53(21.0 \%)$ & $142(56.3 \%)$ & $31(12.3 \%)$ & $26(10.3 \%)$ & o $(0 \%)$ & $\begin{array}{l}3.88 \pm \\
0.857\end{array}$ \\
\hline $\begin{array}{l}\text { 11. I find it hard to } \\
\text { say how I feel about } \\
\text { other people }\end{array}$ & $39(15 \cdot 5 \%)$ & $137(54.4 \%)$ & $31(12.3 \%)$ & $45(17 \cdot 9 \%)$ & o $(0 \%)$ & $\begin{array}{l}3.67 \pm \\
0.943\end{array}$ \\
\hline $\begin{array}{c}\text { 12. Other people tell } \\
\text { me that I should talk } \\
\text { more about how I } \\
\text { feel inside }\end{array}$ & $46(18.3 \%)$ & $139(55.2 \%)$ & $38(15.1 \%)$ & $29(11.5 \%)$ & o $(0 \%)$ & $\begin{array}{l}3.80 \pm \\
0.870\end{array}$ \\
\hline $\begin{array}{l}\text { 13. I don't know } \\
\text { what's going on } \\
\text { inside me }\end{array}$ & $15(6.0 \%)$ & $185(73.4 \%)$ & $32(12.7 \%)$ & $20(7 \cdot 9 \%)$ & o $(0 \%)$ & $\begin{array}{l}3 \cdot 77 \pm \\
0.674\end{array}$ \\
\hline $\begin{array}{c}\text { 14. I often don't } \\
\text { know why I am angry }\end{array}$ & $29(11.5 \%)$ & $174(69.0 \%)$ & $41(16.3 \%)$ & $8(3.2 \%)$ & $\mathrm{o}(\mathrm{o} \%)$ & $\begin{array}{l}3.89 \pm \\
0.628\end{array}$ \\
\hline
\end{tabular}




\begin{tabular}{|c|c|c|c|c|c|c|}
\hline $\begin{array}{l}\text { 15. I prefer talking to } \\
\text { people about } \\
\text { everyday things, } \\
\text { rather than about } \\
\text { how they feel }\end{array}$ & $41(16.3 \%)$ & $162(64.3 \%)$ & $36(14 \cdot 3 \%)$ & $12(4.8 \%)$ & $1(4.0 \%)$ & $3.91 \pm 0.725$ \\
\hline $\begin{array}{l}\text { 16. I prefer watching } \\
\text { funny television } \\
\text { programmes, rather } \\
\text { than films that tell a } \\
\text { story about other } \\
\text { people's problems }\end{array}$ & $93(36.9 \%)$ & $116(46.0 \%)$ & $26(10.3 \%)$ & $8(3.2 \%)$ & o $(0 \%)$ & $4.27 \pm 0.818$ \\
\hline $\begin{array}{l}\text { 17. It is difficult for } \\
\text { me to say how I } \\
\text { really feel inside, } \\
\text { even to my best } \\
\text { friend }\end{array}$ & $55(21.8 \%)$ & $160(63.5 \%)$ & $36(14.3 \%)$ & $1(4.0 \%)$ & o $(0 \%)$ & $4.07 \pm 0.611$ \\
\hline $\begin{array}{c}\text { 18. I can feel close to } \\
\text { someone, even when } \\
\text { we are sitting still } \\
\text { and not saying } \\
\text { anything }\end{array}$ & $20(7 \cdot 9 \%)$ & $174(69.0 \%)$ & $41(16.3 \%)$ & $17(6.7 \%)$ & o $(\mathrm{o} \%)$ & $3.78 \pm 0.683$ \\
\hline $\begin{array}{l}\text { 19. Thinking about } \\
\text { how I feel, helps me } \\
\text { when I want to do } \\
\text { something about my } \\
\text { problems }\end{array}$ & $33(13.1 \%)$ & $142(56.3 \%)$ & $48(19.0 \%)$ & $25(9.9 \%)$ & $4(1.6 \%)$ & $3.69 \pm 0.878$ \\
\hline $\begin{array}{l}\text { 20. When I have to } \\
\text { concentrate on a film } \\
\text { to understand the } \\
\text { story, I enjoy the } \\
\text { film much less }\end{array}$ & $97(38.5 \%)$ & $129(51.2 \%)$ & $22(8.7 \%)$ & $4(1.6 \%)$ & $\mathrm{o}(\mathrm{o} \%)$ & $4.27 \pm 0.683$ \\
\hline
\end{tabular}

Table 1. Frequency Distribution of Respondent Regarding Alexithymia

trial of brief psychoanalytic psychotherapy in patients with functional dyspepsia. Asian J Psychiatr 2013;6(3): 228-34.

3. Faramarzi M, Kheirkhah F, Shokri-Shirvani J, Mosavi $\mathrm{S}$, Zarini S. Psychological factors in patients with peptic ulcer and functional dyspepsia. Caspian J Inter Med. 2014;5(2):71-6.

4 Faramarzi M, Shokri-Shirvani J, Kheirkhah F. The role of psychiatric symptoms, alexithymia, and maladaptive defenses in patients with functional dyspepsia. Indian J Med Sci. 2012;66(1-2):40-8.

5 Cox BJ, Swinson RP, Shulman ID, Bourdeau D. Alexithymia in panic disorder and social phobia. Compr. Psychiatry 1995; 36(3):195-8.

6. Taylor GJ. Bagby RM. New Trends in Alexithymia Research. Psychother Psychosom 2004; 73(2):68-77.

7. Bagby A, Taylor RM, Parker GJ, Dickens SE. The development of the Toronto Structured Interview for Alexithymia: Item selection, factor structure, reliability and concurrent validity. Psychotherapy and Psychosomatics 2006;75:25-39. doi:10.1159/000089224. 8. Faramarzi M, Salmalian H. Association of psychologic and non-psychologic factors with primary dysmenorrhea. Iran Red Crescent Med J 2014; 16(8):e19307.

9.Parker JDA, Austin EJ, Hogan HJ, Wood LM, Bond BJ. Alexithymia and academic success: examining the transition from high school to university. Pers Individ Differ 2005; 38(6):1257-67.

10 Sepahvand E, Zakiei A, Rafieian K, Roumani S, Komasi S, Reshadat S. The intervening role of alexithymia in the relationship between attachment styles and test anxiety among gifted high school students. Korean J Fam Med. 2015; 36(4):174-9.

11. Delavar MA, Salmalian H, Faramarzi M. Pasha H, Bakhtiari A, Nikpour M, Ledari FM. Using the objective structured clinical examinations in undergraduate midwifery students. J Med Life 2013;6(1):76-9. 


\begin{tabular}{|c|c|c|c|c|c|c|c|}
\hline & $\mathbf{G P}$ & $\mathbf{N}$ & Mean & $\begin{array}{l}\text { Std. } \\
\text { Deviati } \\
\text { on }\end{array}$ & $\begin{array}{l}\text { Std. Error } \\
\text { Mean }\end{array}$ & $\begin{array}{l}\text { Mean } \\
\text { differen } \\
\text { ce }\end{array}$ & P value \\
\hline \multirow{2}{*}{$\begin{array}{l}\text { I I am often confused about the way I am } \\
\text { feeling inside }\end{array}$} & Ayurveda & 113 & 3.45 & .802 & .075 & \multirow{2}{*}{$\begin{array}{l}-.016 \\
-.016\end{array}$} & \multirow[t]{2}{*}{$0.5^{* *}$} \\
\hline & Dental & 139 & 3.47 & .837 & .071 & & \\
\hline \multirow[t]{2}{*}{2 I find it difficult to say how I feel inside } & Ayurveda & 113 & 3.45 & .802 & .075 & \multirow{2}{*}{$\begin{array}{l}-.016 \\
-.016\end{array}$} & \multirow[t]{2}{*}{$0.5^{* *}$} \\
\hline & Dental & 139 & $3 \cdot 47$ & .837 & .071 & & \\
\hline \multirow{2}{*}{$\begin{array}{l}3 \text { I feel things in my body that even doctors } \\
\text { don't understand }\end{array}$} & Ayurveda & 113 & $3 \cdot 33$ & .871 & .082 & \multirow{2}{*}{$\begin{array}{r}-.004 \\
-.004\end{array}$} & \multirow[t]{2}{*}{$0.4^{* *}$} \\
\hline & Dental & 139 & 3.33 & .904 & .077 & & \\
\hline \multirow[t]{2}{*}{4 I can easily say how I feel inside } & Ayurveda & 113 & $3 \cdot 70$ & .693 & .065 & \multirow{2}{*}{$\begin{array}{r}.059 \\
.059\end{array}$} & \multirow[t]{2}{*}{$0.02^{*}$} \\
\hline & Dental & 139 & 3.64 & .851 & .072 & & \\
\hline \multirow{2}{*}{$\begin{array}{l}5 \text { When I have a problem, I want to know } \\
\text { where it comes from and not just talk about } \\
\text { it }\end{array}$} & Ayurveda & 113 & 3.62 & .783 & .074 & \multirow{2}{*}{$\begin{array}{l}-.107 \\
-.107\end{array}$} & \multirow[t]{2}{*}{$0.1^{* *}$} \\
\hline & Dental & 139 & $3 \cdot 73$ & .962 & .082 & & \\
\hline \multirow{2}{*}{$\begin{array}{l}6 \text { When I am upset, I don't know if I am sad, } \\
\text { scared or angry }\end{array}$} & Ayurveda & 113 & 3.69 & .733 & .069 & \multirow{2}{*}{$\begin{array}{l}.144 \\
.144\end{array}$} & \multirow[t]{2}{*}{$0.005^{*}$} \\
\hline & Dental & 139 & $3 \cdot 55$ & .878 & .074 & & \\
\hline \multirow{2}{*}{$\begin{array}{l}7 \mathrm{I} \text { am often puzzled by things that I feel in } \\
\text { my body }\end{array}$} & Ayurveda & 113 & 3.24 & 1.002 & .094 & \multirow{2}{*}{$\begin{array}{l}-.121 \\
-.121\end{array}$} & \multirow[t]{2}{*}{$0.6^{* *}$} \\
\hline & Dental & 139 & 3.36 & .985 & .084 & & \\
\hline \multirow[t]{2}{*}{$\begin{array}{l}8 \text { I'd rather wait and see what happens, } \\
\text { instead of thinking about why things happen }\end{array}$} & Ayurveda & 113 & 3.21 & .796 & .075 & \multirow{2}{*}{$\begin{array}{l}-.054 \\
-.054\end{array}$} & \multirow[t]{2}{*}{$0.02^{*}$} \\
\hline & Dental & 139 & 3.27 & .873 & .074 & & \\
\hline \multirow[t]{2}{*}{$\begin{array}{l}\text { 9 Sometimes I can't find the words to say how } \\
\text { I feel inside }\end{array}$} & Ayurveda & 113 & 3.67 & .700 & .066 & \multirow{2}{*}{$\begin{array}{l}-.248 \\
-.248\end{array}$} & \multirow[t]{2}{*}{$0.9^{* *}$} \\
\hline & Dental & 139 & 3.92 & .826 & .070 & & \\
\hline \multirow[t]{2}{*}{$\begin{array}{l}10 \text { It is important to understand how you feel } \\
\text { inside }\end{array}$} & Ayurveda & 113 & 3.95 & .789 & .074 & \multirow{2}{*}{.120} & $0.06^{* *}$ \\
\hline & Dental & 139 & 3.83 & .908 & .077 & & \\
\hline $\begin{array}{l}\text { II I find it hard to say how I feel about other } \\
\text { people }\end{array}$ & Ayurveda & 113 & 3.66 & .951 & .089 & -.020 & $0.8^{* *}$ \\
\hline & Dental & 139 & 3.68 & .941 & .080 & -.020 & \\
\hline 12 Other people tell me that I should talk & Ayurveda & 113 & 4.11 & .783 & .074 & $.55^{2}$ & $0.1^{* *}$ \\
\hline more about how I feel inside & Dental & 139 & $3 \cdot 55$ & .861 & .073 & .552 & \\
\hline 13 I don't know what's going on inside me & Ayurveda & 113 & 3.82 & .671 & .063 & .089 & $0.5^{* *}$ \\
\hline & Dental & 139 & $3 \cdot 73$ & .676 & .057 & .089 & \\
\hline 14 I often don't know why I am angry & Ayurveda & 113 & $3 \cdot 97$ & .589 & .055 & .153 & $0.1^{* *}$ \\
\hline & Dental & 139 & 3.82 & .651 & .055 & .153 & \\
\hline 15 I prefer talking to people about everyday & Ayurveda & 113 & 4.06 & .631 & .059 & .271 & $0.01^{*}$ \\
\hline things, rather than about how they feel & Dental & 139 & $3 \cdot 79$ & .775 & .066 & .271 & \\
\hline $\begin{array}{l}16 \text { I prefer watching funny television } \\
\text { programmes, rather than films that tell a }\end{array}$ & Ayurveda & 113 & 4.17 & .833 & .078 & -.192 & $0.2^{* *}$ \\
\hline story about other people's problems & Dental & 139 & $4 \cdot 36$ & .799 & .068 & -.192 & \\
\hline
\end{tabular}




\begin{tabular}{|c|c|c|c|c|c|c|c|}
\hline \multirow{2}{*}{$\begin{array}{l}17 \text { It is difficult for me to say how I really feel } \\
\text { inside, even to my best friend }\end{array}$} & Ayurveda & 113 & 3.96 & .706 & .066 & \multirow{2}{*}{$\begin{array}{l}-.186 \\
-.186\end{array}$} & \multirow[t]{2}{*}{$0.01^{*}$} \\
\hline & Dental & 139 & 4.15 & .510 & .043 & & \\
\hline \multirow{2}{*}{$\begin{array}{l}18 \text { I can feel close to someone, even when we } \\
\text { are sitting still and not saying anything }\end{array}$} & Ayurveda & 113 & 3.78 & .741 & .070 & \multirow{2}{*}{$\begin{array}{l}-.005 \\
-.005\end{array}$} & \multirow[t]{2}{*}{$0.006^{*}$} \\
\hline & Dental & 139 & 3.78 & .634 & .054 & & \\
\hline \multirow{2}{*}{$\begin{array}{l}19 \text { Thinking about how I feel, helps me when } \\
\text { I want to do something about my problems }\end{array}$} & Ayurveda & 113 & 3.80 & .888 & .084 & \multirow{2}{*}{$\begin{array}{r}.185 \\
.185\end{array}$} & \multirow[t]{2}{*}{$0.3^{* *}$} \\
\hline & Dental & 139 & 3.61 & .864 & .073 & & \\
\hline \multirow{2}{*}{$\begin{array}{l}20 \text { When I have to concentrate on a film to } \\
\text { understand the story, I enjoy the film much } \\
\text { less }\end{array}$} & Ayurveda & 113 & 4.07 & .678 & .064 & \multirow{2}{*}{$\begin{array}{l}-.354 \\
-.354\end{array}$} & \multirow[t]{2}{*}{$0.002^{*}$} \\
\hline & Dental & 139 & 4.42 & .648 & .055 & & \\
\hline
\end{tabular}

Table 2. Inter Group Comparison of Respondents According to Course of Study

12. Bataineh MZ. Academic stress among undergraduate students: the case of education faculty at King Saud university. Int Interdiscip J Educ. 2013; 2(1):82-8.

13. Twenge JM, Gentile B, DeWall CN, Ma D, Lacefield K, Schurtz DR. Birth cohort increases in psychopathology among young Americans, 1938-2007: a cross-temporal metaanalysis of the MMPI. Clin Psychol Rev. 2010; 30(2):145-54.
14. Faramarzi M, Kheirkhah F, Azadfrouz S. Psychological predictors of premenstrual syndrome. Int Neuropsychiatr Dis J. 2014; 2(6):368-81.

15 Kalinin VV, Zemlyanaya AA, Krylov OE, Zheleznova EV. Handedness, alexithymia, and focus laterality as risk factors for psychiatric comorbidity in patients with epilepsy. Epilepsy Behav 2010; 17(3):389-4.

16. Lee SA, Lee SM, No YJ. Factors contributing to depression in patients with epilepsy. Epilepsia 2010; 51(7):1305-8.
Source of support: Nil, Conflict of interest: None declared

\section{Cite this article as:}

Chaudhary D, Meena A. Role of Alexithymia in Predicting Self-Efficacy in Academic Students of a North Indian Institution: A Cross Sectional Study. Int Healthc Res J. 2019;3(8):275-280. https://doi.org/10.26440/IHRJ/0308.11202

\section{AUTHOR AFFILIATIONS: $\left({ }^{*}\right.$ Corresponding Author)}

1. PG Student, Department of Public Health Dentistry, D.J. College of Dental Sciences and Research, Modinagar, UP, India

2. *Junior Resident, Department of Dentistry, GTB Hospital, New Delhi, 110095 